\title{
Contaminantes ambientais: efeitos dos microplásticos em organismos aquáticos e
}

\section{terrestres}

\author{
Environmental contaminants: effects of microplastics on aquatic and terrestrial organisms \\ Contaminantes ambientales: efectos de los microplásticos sobre organismos acuáticos y terrestres
}

Recebido: 03/06/2021 | Revisado: 10/06/2021 | Aceito: 17/06/2021 | Publicado: 02/07/2021

Ducilene do Carmo da Silva

ORCID: https://orcid.org/0000-0001-8074-7486

Universidade Federal do Tocantins, Brasil

E-mail:ducycarmo045@gmail.com

Hellem Antonia Guedes Vieira

ORCID: https://orcid.org/0000-0002-7871-5554

Universidade Federal do Tocantins, Brasil

E-mail: hellemguedes@outlook.com

Vitória Silva Rolim

ORCID: https://orcid.org/0000-0003-3495-6740

Universidade Federal do Tocantins, Brasil

E-mail: vitoriarolim20@gmail.com

Wanderson Ferreira da Silva

ORCID: https://orcid.org/0000-0002-5363-5099

Universidade Federal do Tocantins, Brasil

E-mail: wandersonqw19@gmail.com

Márcio Guimarães de Sousa

ORCID: https://orcid.org/0000-0003-1064-4793

Universidade Federal do Tocantins, Brasil

E-mail: marcio.sousa-@hotmail.com

Marcelo Gustavo Paulino

ORCID: https://orcid.org/0000-0003-0464-6848

Universidade Federal do Tocantins, Brasil

E-mail: marcelopaulino@uft.edu.br

Wagner dos Santos Mariano

ORCID: https://orcid.org/0000-0003-0225-6889

Universidade Federal do Tocantins, Brasil

E-mail: wagnermariano@uft.edu.br

\begin{abstract}
Resumo
Intensificada no século XVIII, a contaminação ambiental tem crescido progressivamente em todo o mundo, gerando de forma radical resíduos tóxicos que proliferam a cada ano. Dentre os poluentes mais preocupantes da atualidade, destacam-se os microplásticos, pequenos pedaços de plásticos inferiores a $5 \mathrm{~mm}$ de diâmetro. $\mathrm{O}$ presente trabalho tem por objetivo realizar um apanhado literário voltado aos impactos ambientais, evidenciando os efeitos dos microplásticos em organismos aquáticos e terrestres. A coleta pelos referenciais teóricos utilizados foi realizada em sites de confiabilidade técnico-científica, com intuito de selecionar artigos científicos, dissertações e e-books que após triados, foram analisados, tabulados e categorizados. Os critérios estabelecidos para escolha do material revisado se deram a partir da leitura do título e do resumo. Após análises dos materiais bibliográficos utilizados neste estudo, ficou claro e perceptível que os microplásticos afetam negativamente de forma direta e/ou indiretamente organismos aquáticos e terrestres, sendo a ingestão a principal via de contaminação biótica.
\end{abstract}

Palavras-chave: Poluentes; Ecotoxicologia, Impactos ambientais.

\section{Abstract}

Intensified in the 18th century, environmental contamination has grown progressively around the world, radically generating toxic waste that proliferates every year. Among the most worrying pollutants of today, we highlight microplastics, small pieces of plastics less than $5 \mathrm{~mm}$ in diameter. The present work aims to carry out a literary survey focused on environmental impacts, showing the effects of microplastic on aquatic and terrestrial organisms. The search for the theoretical references used was carried out on websites of complete reliability, and scientific articles, dissertations and e-books were pre-selected for analysis. The criteria established for choosing the revised material 
came from reading the title and abstract. Given the analyzed literature, it was clearly noticeable that microplastics negatively affect directly and / or indirectly aquatic and terrestrial organisms, with ingestion being the main route of biotic contamination.

Keywords: Pollutants; Ecotoxicology; Envoronmental impacts.

\section{Resumen}

Intensificado en el siglo XVIII, la contaminación ambiental ha crecido progresivamente en todo el mundo, generando de manera radical desechos tóxicos que proliferan cada año. Entre los contaminantes más preocupantes de la actualidad, destacamos los microplásticos, pequeños trozos de plástico de menos de $5 \mathrm{~mm}$ de diámetro. El presente trabajo tiene como objetivo realizar una encuesta literaria centrada en los impactos ambientales, mostrando los efectos de los microplásticos en los organismos acuáticos y terrestres. La recopilación por las referencias teóricas utilizadas se realizó en sitios de confiabilidad técnico-científica, con el fin de seleccionar artículos científicos, disertaciones y libros electrónicos que, luego de ser revisados, fueron analizados, tabulados y categorizados. Los criterios establecidos para la elección del material revisado se basaron en la lectura del título y del resumen. Tras analizar los materiales bibliográficos utilizados en este estudio, quedó claro y notorio que los microplásticos afectan de forma negativa directa y / o indirecta a los organismos acuáticos y terrestres, siendo la ingestión la principal vía de contaminación biótica.

Palabras clave: Contaminantes; Ecotoxicología; Impactos ambientales.

\section{Introdução}

Intensificada no século XVIII, a contaminação ambiental tem crescido progressivamente em todo o mundo, gerando de forma radical resíduos tóxicos que proliferam a cada ano (Peres et al., 2001). Com isso, os problemas ambientais advindos desses resíduos têm alertado para a necessidade urgente da preservação dos recursos naturais (Neves et al., 2016).

Os contaminantes ambientais podem ser substâncias naturais ou produtos industrializados, podendo ser manufaturados ou derivados de subprodutos produzidos não intencionalmente pela atividade antrópica (Peres et al., 2001). Os plásticos se destacam como um dos principais poluentes da atualidade, o qual tem sido motivo de preocupação ambiental devido à grande quantidade de resíduos que são gerados (Olivatto et al., 2018). Além da preocupação com detritos plásticos maiores, outra categoria vem ganhando destaque nos últimos anos, os chamados microplásticos (MPs), caracterizados como pequenos pedaços de plásticos inferiores a $5 \mathrm{~mm}$ de diâmetro (Lucio et al., 2019).

Os microplásticos podem ser classificados em primários e secundários. Os MPs primários são caracterizados como aqueles já fabricados em escala micro, com a finalidade de serem utilizados principalmente em produtos de limpeza facial e cosméticos. Já os secundários, são pequenos fragmentos plásticos provenientes da quebra de plásticos maiores em ambientes aquáticos e terrestres (Cole et al., 2011). O impacto ambiental provindo dos microplásticos se deve a duas razões principais: (1) seu tamanho pequeno facilita a internalização de detritos pela biota, o que consequentemente resulta em bioacumulação na cadeia alimentar; (2) os MPs tem a capacidade de adsorver poluentes orgânicos persistentes (POPs) em suas superfícies (Anbumani et al., 2018). A introdução de MPs nos ecossistemas aquáticos e terrestres, ocorrem de forma direta através de escoamento e/ou decomposição por intemperismo de detritos meso e microplásticos, podendo ocasionar efeitos ecotoxicológicos e por vezes deletérias em muitos organismos (Andrady, 2011; Mendoza et al., 2018).

Além dos efeitos tóxicos do microplástico na biota aquática, os organismos terrestres também estão suscetíveis a tal poluição, através da inalação e ingestão de alimentos contaminados (Olivatto et al., 2018). Os MPs têm a capacidade de persistir no ambiente e ser transferido de um nível trófico para outro (processo denominado de bioacumulação), causando danos em todo o ecossistema (de Paula Duarte, 2019).

Portanto, o objetivo deste trabalho é realizar uma revisão de literatura centrada nos impactos causados por poluentes ambientais, evidenciando os efeitos do microplástico em organismos aquáticos e terrestres. 


\section{Metodologia}

Este estudo é de cunho exploratório e abordagem qualitativa, baseado em revisão bibliográfica, cujo levantamento de dados se deu através da análise de trabalhos publicados entre os anos de 2003 a 2021 em idioma português e inglês. Para busca de dados bibliográficos, considerou-se artigos que apresentassem no tema, termos como: "effects of microplastic", "pollution microplastic", "Microplastic in environment" e "microplásticos nos ecossistemas".

Os artigos analisados levam em consideração, exclusivamente, os efeitos deletérios causados por micropartículas plásticas em organismos aquáticos e terrestres visto que, ainda é um contaminante emergente de preocupação mundial e com efeitos ainda poucos estudados. Uma pesquisa de caráter exploratória tem por objetivo aprimorar e/ou construir hipóteses, validar instrumentos e proporcionar maior familiaridade com o problema em estudo (Gerhardt \& Silveira., 2009). Tal pesquisa, remete-se à primeira etapa de um estudo mais amplo, visando a um apanhado geral sobre temas comumente pouco explorados (Franco \& Dantas, 2017).

\section{Resultados e Discussão}

O levantamento bibliográfico baseou-se na análise de 52 artigos científicos e uma única dissertação disponíveis na plataforma do Google Scholar e Periódicos Capes. Os artigos analisados foram distribuídos nas seguintes revistas científicas: Marine pollution (6); Water research (1); Global change biology (2); Current Opinion in Environmental Science \& Health (3); Environmental toxicology and pharmacology (1); Environmental Pollution (13); Environmental science \& technology (6); Conexão Ciência (1); Revista Virtual de Química (1); Ecotoxicology and environmental safety (1); Science of The Total Environment (7); Ecological indicators (1); Scientific reports (2); Plos one (1); Enciclopédia Biosfera (1); TrAC Trends in Analytical Chemistry (1), ICES Journal of Marine Science (1); Conservation biology (1) e Journal of Marine Biology (1).

Diante disso, percebe-se que a revista que mais recebeu publicações sobre o impacto dos microplásticos em organismos aquáticos e terrestres entre os anos 2003 a 2021 foi a Environmental Pollution, com 13 artigos publicados, mostrando a relevância desse periódico para a área ecotoxicologia dos MPs. Além disso, observou-se que a grande maioria dos trabalhos que tratam sobre o tema foram artigos científicos, sendo encontrada apenas uma única dissertação.Acerca do idioma, encontrou-se trabalhos tanto em Inglês quanto em Português, sendo a minoria produzida nesta segunda língua ( 4 artigos e 1 dissertação), o que aponta para uma necessidade na elaboração de mais obras neste respectivo idioma. Por fim, nas seguintes seções temos os principais pontos que os autores consultados abordam dentro dos efeitos dos microplásticos em organismos aquáticos e terrestres.

\section{Microplásticos no ambiente}

A poluição ambiental ocasionada por resíduos plásticos tem crescido demasiadamente nos últimos anos, e como resultado de sua degradação, temos uma grande quantidade de acúmulo de micropartículas plásticas no ambiente. Tais contaminantes têm gerado constante preocupação, pois são vistos como uma ameaça ambiental, uma vez que podem ser ingeridos pela biota e, consequentemente gerar danos físicos e fisiológicos em mamíferos, peixes, pássaros dentro outros animais que também podem ser afetados de forma direta ou indiretamente (Ogonowski et al., 2018).

Devido ao tamanho reduzido de tais partículas, a identificação e caracterização torna-se um desafio. Quando disponíveis no ar, os MPs podem somente ser observados com a utilização de equipamentos de ampliação (microscópios e lupas), o que acaba dificultando ainda mais sua amostragem e caracterização (de Paula Duarte, 2019). Apesar do alerta científico sobre os problemas decorrentes da poluição microplástica, a quantidade desses resíduos gerados continua aumentando significativamente no meio ambiente, e a produção plástica industrial permanece crescendo diariamente (Oliveira et al., 2013). 


\section{Microplásticos em ambientes aquáticos}

Estudos direcionados aos efeitos ecotoxicológicos dos microplásticos (MPs) em corpos hídricos de água doce e ambiente marinho ainda são limitados (Olivatto et al., 2018). Entretanto, a presença de MPs nesses meios tem chamado a atenção de pesquisadores ambientalistas acerca das consequências que essas micropartículas podem causar à biota aquática.

A ingestão é a interação mais provável com os MPs para muitos organismos aquáticos. Quando ingeridas, essas micropartículas podem bloquear o intestino, diminuir os níveis de hormônios esteróides, interferir na reprodução, impedir o crescimento e reduzir a absorção de nutrientes pelo organismo (Oliveira et al., 2013; Gallagher et al., 2016). Neste contexto, supõe-se que essa probabilidade de impacto na reprodução da biota, pode resultar em diminuição da prole, sugerindo-se que tal efeito pode até mesmo acarretar na extinção de muitas espécies.

Os microplásticos podem ser ingeridos de forma direta por animais filtradores ou acidentalmente, sendo confundidos com alimentos (Gallagher et al., 2016; Avio et al., 2015). Pesquisas voltadas aos efeitos dos microplásticos na biota aquática, tem particular interesse em estudar animais bivalves, pois durante sua atividade alimentar, filtram um grande volume de água, acarretando em exposição direta aos MPs disponíveis na coluna d'água (Foley et al., 2018; Li et al., 2015 ). De acordo com os estudos de Browne et al. (2008) e Von Moos et al. (2012) com mexilhões in vivo, observaram que micropartículas plásticas de poliestireno e polietileno são capazes de translocar-se da cavidade intestinal para a corrente sanguínea, entrar nas células e provocar efeitos negativos a nível celular e tecidual. Sabendo que detritos plásticos têm a capacidade de acumular-se nesses organismos, o consumo destes por inteiro, ignorando a remoção do intestino, podem ser uma rota de exposição para os ser humano (Cho et al., 2021).

A ameaça microplástica também assola espécies em conservação, como as tartarugas marinhas, que diariamente estão sujeitas a uma série de estressores antropogênicos (Guzzetti et al., 2018). Esses animais são expostos a tais contaminantes através da ingestão direta ou indireta de detritos plásticos (Hoarau et al., 2014; Schuyler et al., 2014; Duncan et al., 2019). A ingestão acidental (direta) pode acontecer quando os micropoluentes plásticos encontram-se misturados ou aderidos a alimentação, enquanto a ingestão indireta ocorre por meio do consumo de presas previamente contaminadas com MPs (Caron et al., 2018; Nelms et al., 2016). Segundo Nelms et al. (2016), como efeito adverso as tartarugas marinhas, essas micropartículas podem causar sérios danos ao seu sistema digestivo, como obstrução do trato intestinal, redução do estímulo alimentar e diminuição da capacidade estomacal, podendo ocasionar desnutrição e, em último instância, levar o indivíduo a morte. O grande acúmulo de microplástico no intestino de tartarugas, pode alterar o comportamento da natação e interferir na flutuabilidade do animal, o que influencia negativamente a atividade predatória e a capacidade de escapar de predadores, além de interferir na taxa de crescimento, fecundidade, fertilidade e sistema imunológico, sendo que, alterações neste último resulta em susceptibilidade a doenças, como por exemplo, a fibropapilomatose (Guzzetti et al., 2018).

Os peixes são organismos amplamente estudados como bioindicador de resposta aos MPs (Khan et al., 2015; Mak et al., 2019; Malafaia et al., 2020). Geralmente, os peixes são contaminados durante a sua atividade alimentar normal, de forma direta ou indireta, no entanto, há outras vias na qual essas partículas se tornam prejudiciais a esses animais (Caixeta et al., 2019; Huang et al., 2020). Muitos peixes se alimentam de organismos planctônicos, e como estratégia alimentar, aspiram grande volume de água para então conseguirem engolir vários itens ao mesmo tempo, no entanto, esta estratégia de captura torna-se prejudicial, uma vez que os detritos plásticos dispersos no meio, e podem ser engolidos acidentalmente durante o ato (Ory et al., 2018).

Embora os peixes tenham paladar bem desenvolvido, muitas espécies ainda não conseguem distinguir de presas comestíveis, resultando na ingestão acidental de detritos plásticos (Ory et al., 2018) Como resultado desta ingestão, os fragmentos microplásticos causam efeitos deletérios aos peixes, podendo danificar ou bloquear o trato gastrointestinal, gerar 
respostas inflamatórias, reduzir o crescimento, gerar danos oxidativos, lesões celulares, desregular o sistema endócrino ou até mesmo suprimir a capacidade de energia do animal (Cole et al., 2011; Horton et al., 2017; Rochman et al., 2014).

Usando o peixe medaka japonesa (Oryzias latipes) como organismo de indicador, Rochman et al. (2013), observou que a exposição do animal a microplásticos de polietileno causou estresse hepático, depleção do glicogênio, vacuolização gordurosa. Acrescentando tais efeitos adversos, o estudo de Lu et al. (2016) expondo peixe-zebra (D. rerio) a micropartículas plásticas de poliestireno, constatou que o contaminante tóxico causou interferência no metabolismo lipídico e energético do animal, além de provocar estresse oxidativo no fígado.

De acordo com o estudo de Oliveira et al. (2013), os MPs de polietileno podem causar reduções significativas na atividade enzimática da acetilcolinesterase - AchE (enzima responsável pela finalização da transmissão dos impulsos nervosos nas sinapses colinérgicas pela hidrólise do neurotransmissor acetilcolina) do peixe gódio (Pomatoschistus microps), juntamente com dano oxidativo, indicando efeitos adversos na neurotransmissão colinérgica, bem como na função nervosa e neuromuscular do peixe. Na natureza, a redução de energia pode limitar consideravelmente a capacidade dos peixes escaparem de predadores e se alimentarem, provavelmente diminuindo sua aptidão individual e aumentando a taxa de mortalidade na população (Guzzetti et al., 2018).

Simmonds (2012) relata que as espécies de cetáceos são susceptíveis a ingestão de detritos plásticos, dando destaque às baleias. A contaminação por MPs ocorre de forma direta, através da ingestão da água durante o processo alimentar de filtração, ou indiretamente por transferência trófica (Fossi et al., 2016; Lusher et al., 2015). Devido ao mecanismo alimentar não seletivo desses animais, há uma alta probabilidade dos microplásticos dispersos na superfície da água serem ingeridos acidentalmente, podendo resultar em danos nos processos digestivos e obstrução do trato gastrointestinal, além da possibilidade de obstrução do aparato de filtragem (Besseling et al., 2015; Fossi et al., 2012; Simmonds., 2012).

Além desta gama de efeitos adversos que os MPs podem causar nos peixes, o processo de biomagnificação também sido motivo de grande preocupação, pois uma vez acumulado no tecido animal, podem ser transferidos para seres de níveis tróficos mais elevados (via cadeia alimentar), incluindo humanos, de modo que as toxinas contidas no peixe contaminado podem ser absorvidas via dieta (Miller et al., 2020; Vethaak \& Leslie, 2016).

\section{Microplásticos em ambientes terrestres}

Os estudos sobre a toxicidade do microplásticos em ecossistemas terrestres ainda são limitados (Jiang et al., 2020). Os detritos plásticos caracterizam-se como um potencial ameaça para a organismos de solo, uma vez que esses micropoluentes podem causar alterações nesse ambiente (de Souza Machado et al., 2018). A ampla distribuição desse material, motivou vários cientistas a estudar e investigar a incidência desses detritos em muitos animais, observando as causas, consequências e respostas de organismos vivos, na presença de tais materiais plásticos (Lucio et al., 2019). Diferentemente da preocupação com a contaminação de tais poluentes nos ambientes aquáticos, os MPs em ecossistemas terrestres têm recebido relativamente pouca atenção, por exemplo no solo a principal via de contaminação de MPs é decorrente de insumos de práticas agrícolas, sobretudo a utilização de lodo de estação de tratamento de esgoto (He et al., 2018). Além disso, águas residuais não tratadas para a irrigação da plantação, também contribuem significativamente para este aumento de micropartículas plásticas no solo (Bläsing \& Amelung, 2018). Outras fontes que corroboram para o acúmulo de MPs nos solos incluem, escoamento de estradas ou áreas urbanas (Rillig et al., 2017).

Com o microplástico presente no solo superficial, vários seres vivos de solo podem contribuir para a movimentação de partículas microplásticas através de ingestão, escavação e aderência, dessa forma estudos com minhocas (Lwanga et al. 2017), colêmbolos (Maab et al., 2017), nematóides (Fueser et al., 2019) e ácaros (Zhu et al., 2018) apontam que esses seres vivos têm possibilidade de transportar micropartículas de PEBD (poliestireno de baixa densidade), PET (tereftalato de 
polietileno), PS (poliestireno) e PVC (policloreto de vinila), respectivamente. Quanto aos efeitos adversos desencadeados pela exposição a microplásticos, Song et al. (2019) e Huerta Lwanga et al. (2016) destacam que, quando essas micropartículas são ingeridas ou se acumulam em animais de solo, pode resultar em dano físico de órgãos e tecidos devido a abrasão com o detrito plástico, desencadeando deste modo, respostas inflamatórias, insuficiência e redução nos níveis de energia.

Esses contaminantes podem chegar ao ser humano através da biomagnificação. O processo de biomagnificação referese ao acúmulo progressivo de substâncias entre níveis tróficos ao longo de cadeias alimentares (Ricklefs., 2003). Com isso, é evidente que tal processo afeta negativamente a cadeia alimentar dos seres vivos, em destaque a saúde humana, o qual pode provocar a redução da quantidade de energia disponível para o crescimento e sucesso reprodutivo (Lucio et al., 2019). Além disso, existem trabalhos que indicam a presença de microplásticos em sais de mesa (Yang et al., 2015), água potável e água doce (Pivokonsky et al., 2018). O estudo dirigido por Yang et al. (2015) indica que as principais frações de microplásticos encontradas em amostra de sal possuem menos de $200 \mu \mathrm{m}$, sendo o microplástico mais comum o tereftalato de polietileno, já em água mineral foram encontradas partículas de microplástico com dimensões abaixo de $20 \mu \mathrm{m}$ (Schymanski et al. 2018).

De acordo com Revel et al. (2018), os MPs podem interagir com os com o ser humano via ingestão de alimentos contaminados, inalação de micropartículas plásticas pelo ar, e também pela exposição térmica. Quando inalados, Prata et al. (2020) relataram que, embora ainda não relatado em humanos, partículas plásticas inaladas podem contribuir para que haja perturbação no sistema imunológico de outros animais. Além disso, Prata (2018) destaca que a inalação de microplásticos podem acometer a saúde humano, gerando implicações no intestino, bem como ocasionar efeitos tóxicos, devido a habilidade de induzir bloqueio intestinal e/ou tecidual, além de fibroses, congestão e inflamação, incidência de doenças respiratórias e cardiovasculares, podendo levar até a formação de câncer pulmonar.

\section{Considerações Finais}

Nesta revisão, notou-se que as micropartículas plásticas, possuem uma alta capacidade de contaminação, tanto em ambientes aquáticos quanto terrestres, e influenciam negativamente a qualidade de vida da biota, com efeitos adversos que podem resultar em sérios danos na fisiologia do animal, principalmente no que se refere a obstrução e bloqueio do trato digestivo.

Em ambiente aquático, notou-se que a principal via de interação dos MPs com os organismos, ocorre por meio da ingestão de detritos plásticos dispersos na água ou acumulado em presas previamente contaminadas, podendo resultar em uma série de danos morfofisiológicos a estes animais. Em meio terrestre, o solo abriga a maior concentração de MPs e causam impactos principalmente a animais deste habitat. Além disso, em animais terrestres, a principal via de interação, ocorre por meio da inalação desses micropoluentes, o que consequentemente pode resultar em uma série de danos aos organismos, como inflamaç̃oes, bloqueio intestinal e doenças respiratórias e cardiovasculares, dentre outras.

Desta forma, percebe-se que as pesquisas voltadas aos impactos dos MPs sob organismos terrestres são encontradas em menor número quando comparadas a amplitude dos estudos com animais de ambientes marinhos e de água doce. Nesta perspectiva, nota-se a necessidade do desenvolvimento de trabalhos futuros voltados aos efeitos ecotoxicológicos dessas micropartículas plásticas em animais do ecossistema terrestre, como por exemplo, em humanos.

\section{Referências}

Anbumani, S., \& Kakkar, P. (2018). Ecotoxicological effects of microplastics on biota: a review. Environmental Science and Pollution Research, 25(15), 14373-14396. 10.1007/s11356-018-1999-x

Andrady, A. L. (2011). Microplastics in the marine environment. Marine pollution bulletin, 62(8), 1596-1605. 10.1016/j.marpolbul.2011.05.030 
Avio, C. G., Gorbi, S., Milan, M., Benedetti, M., Fattorini, D., d'Errico, G., Pauletto, M., Bargelloni, L., \& Regoli, F. (2015). Pollutants bioavailability and toxicological risk from microplastics to marine mussels. Environmental pollution, 198, 211-222. 10.1016/j.envpol.2014.12.021

Besseling, E., Foekema, E. M., Van Franeker, J. A., Leopold, M. F., Kühn, S., Rebolledo, E. B., Hebe, E., Mielke, L., Ijzer, J., Kamminga, P., \& Koelmans, A. A. (2015). Microplastic in a macro filter feeder: humpback whale Megaptera novaeangliae. Marine pollution bulletin, 95(1), $248-252$. 10.1016/j.marpolbul.2015.04.007

Bläsing, M., Amelung, W. (2018). Plastics in soil: Analytical methods and possible sources. Science of the Total Environment,612, 422-435. 10.1016/j.scitotenv.2017.08.086

Browne, M. A., Dissanayake, A., Galloway, T. S., Lowe, D. M., \& Thompson, R. C. (2008). Ingested microscopic plastic translocates to the circulatory system of the mussel, Mytilus edulis (L.). Environmental science \& technology, 42(13), 5026-5031. 10.1021/es800249a.

Caixeta, D., Caixeta, F. C., \& Menezes Filho, F. (2018). Nano e microplásticos nos ecossistemas: Impactos ambientais e efeitos sobre os organismos. ENCICLOPÉDIA BIOSFERA, 15(27). 10.18677/EnciBio_2018A9

Caron, A. G., Thomas, C. R., Berry, K. L., Motti, C. A., Ariel, E., \& Brodie, J. E. (2018). Ingestion of microplastic debris by green sea turtles (Chelonia mydas) in the Great Barrier Reef: Validation of a sequential extraction protocol. Marine Pollution Bulletin, 127, 743-751. 10.1016/j.marpolbul.2017.12.062

Cho, Y., Shim, W. J., Jang, M., Han, G. M., \& Hong, S. H. (2021). Nationwide monitoring of microplastics in bivalves from the coastal environment of Korea. Environmental Pollution, 270, 116175. 10.1016/j.envpol.2020.116175

Cole, M., Lindeque, P., Halsband, C., \& Galloway, T. S. (2011). Microplastics as contaminants in the marine environment: a review. Marine pollution bulletin, 62(12), 2588-2597. 10.1016/j.marpolbul.2011.09.025

de Souza Machado, A. A., Kloas, W., Zarfl, C., Hempel, S., \& Rillig, M. C. (2018). Microplastics as an emerging threat to terrestrial ecosystems. Global change biology, 24(4), 1405-1416. 10.1111/gcb.14020

de Paula Duarte, J. (2019). Caracterização de microplástico suspenso no ar na unidade de separação de resíduos, LIPOR. Dissertação (Mestrado em Ciências e Tecnologia do Ambiente - Riscos: Avaliação e Gestão Ambiental). Faculdade de Ciências. Universidade do Porto. https://repositorioaberto.up.pt/bitstream/10216/125681/2/378489.pdf

Duncan, E. M., Broderick, A. C., Fuller, W. J., Galloway, T. S., Gosfrey, M. H., Hamann, M., Limpus, C. J., Lindeque, P. K., Mayes, A. G., Omeyer, L. C. M., Santillo, D., Snape, R. T. E., \& Godley, B. J. (2019). Microplastic ingestion ubiquitous in marine turtles. Global change biology, 25(2), 744-752. $10.1111 / \mathrm{gcb} .14519$

Foley, C. J., Feiner, Z. S., Malinich, T. D., \& Höök, T. O. (2018). A meta-analysis of the effects of exposure to microplastics on fish and aquatic invertebrates. Science of the total environment, 631, 550-559. 10.1016/j.scitotenv.2018.03.046

Fossi, M. C., Panti, C., Guerranti, C., Coppola, D., Giannetti, M., Marsili, L., \& Minutoli, R. (2012). Are baleen whales exposed to the threat of microplastics? A case study of the Mediterranean fin whale (Balaenoptera physalus). Marine Pollution Bulletin, 64(11), 2374-2379. 10.1016/j.marpolbul.2012.08.013

Fossi, M. C., Marsili, L., Baini, M., Giannetti, M., Coppola, D., Guerranti, C., Caliani, I., Minutoli, R., Lauriano, G., Finoia, M. G., Rubegni, F., Panigada, S., Bérubé, M., Ramírez, J. U., \& Panti, C. (2016). Fin whales and microplastics: the Mediterranean Sea and the Sea of Cortez scenarios. Environmental Pollution, 209, 68-78. 10.1016/j.envpol.2015.11.022

Franco, M. V. A., \& Dantas, O. M. A. N. A. (2017). Pesquisa exploratória: Aplicando instrumentos de gerações de dados-observação, questionário e entrevista. In EDUCERE - Congresso Nacional de Educação (Vol. 8). https://educere.bruc.com.br/arquivo/pdf2017/25001_13407.pdf

Fueser, H., Mueller, M. T., Weiss, L., Höss, S., \& Traunspurger, W. (2019). Ingestion of microplastics by nematodes depends on feeding strategy and buccal cavity size. Environmental Pollution, 255, 113227. 10.1016/j.envpol.2019.113227

Gallagher, A., Rees, A., Rowe, R., Stevens, J., \& Wright, P. (2016) Microplastic in the Solent estuarine complex, UK: na initial assessment. Marine Pollution Bulletin. 102, 2, 243-249. 10.1016/j.marpolbul.2015.04.002

Gerhardt, T. E., Silveira, D. T. (2009). Métodos de pesquisa. Porto Alegre; Editora da UFRGS. https://books.google.com.br/books?id=dRuzRyEIzmkC\&lpg=PA9\&ots=93Q90WknKG\&dq=Gerhardt\%2C\%20T.\%20E.\%2C\%20Silveira\%2C\%20D.\%20T. \%20(2009).\%20M\%C3\%A9todos\%20de\%20pesquisa.\%20Porto\%20Alegre\%3B\%20Editora\%20da\%20UFRGS.\&lr\&hl=pt-

$\mathrm{BR} \& \mathrm{pg}=\mathrm{PA} 6 \# \mathrm{v}=$ onepage $\& \mathrm{q} \& \mathrm{f}=$ false

Guzzetti, E., Sureda, A., Tejada, S., \& Faggio, C. (2018). Microplastic in marine organism: Environmental and toxicological effects. Environmental toxicology and pharmacology, 64, 164-171. 10.1016/j.etap.2018.10.009

He, D., Luo, Y., Lu, S., Liu, M., Song, Y., \& Lei, L. (2018). Microplastics in soils: analytical methods, pollution characteristics and ecological risks. TrAC Trends in Analytical Chemistry, 109, 163-172. 10.1016/j.trac.2018.10.006

Hoarau, L., Ainley, L., Jean, C., \& Ciccione, S. (2014). Ingestion and defecation of marine debris by loggerhead sea turtles, Caretta caretta, from by-catches in the South-West Indian Ocean. Marine Pollution Bulletin, 84(1-2), 90-96. 10.1016/j.marpolbul.2014.05.031

Horton, A. A., Walton, A., Spurgeon, D. J., Lahive, E., \& Svendsen, C. (2017). Microplastics in freshwater and terrestrial environments: Evaluating the current understanding to identify the knowledge gaps and future research priorities. Science of the total environment, v. 586, p. 127-141. 10.1016/j.scitotenv.2017.01.190

Huang, J. S., Koongolla, J. B., Li, H. X., Lin, L., Pan, Y. F., Liu, S. He, W. H., Maharana, D., \& Xu, X. R. (2020). Microplastic accumulation in fish from Zhanjiang mangrove wetland, South China. Science of The Total Environment, 708, 134839. 10.1016/j.scitotenv.2019.134839 
Huerta Lwanga, E., Gertsen, H., Gooren, H., Peters, P., Salánki, T., Van Der Ploeg, M., Besseling, E., Koelmans, A. A., \& Geissen, V. (2016). Microplastics in the terrestrial ecosystem: implications for Lumbricus terrestris (Oligochaeta, Lumbricidae). Environmental science \& technology, 50(5), $2685-2691$. 10.1021/acs.est.5b05478

Jiang, X., Chang, Y., Zhang, T., Qiao, Y., Klobučar, G., \& Li, M. (2020). Toxicological effects of polystyrene microplastics on earthworm (Eisenia fetida). Environmental Pollution, 259, 113896. 10.1016/j.envpol.2019.113896

Khan, F. R., Syberg, K., Shashoua, Y., \& Bury, N. R. (2015). Influence of polyethylene microplastic beads on the uptake and localization of silver in zebrafish (Danio rerio). Environmental pollution, 206, 73-79. 10.1016/j.envpol.2015.06.009

Li, J., Yang, D., Li, L., \& Jabeen, K., Shi, H. (2015). Microplastics in commercial bivalves from China. Environmental pollution, 207, 190-195. 10.1016/j.envpol.2015.09.018

Lu, Y., Zhang, Y., Deng, Y., Jiang, W., Zhao, Y., Geng, J., Ding, L., \& Ren, H. (2016). Uptake and accumulation of polystyrene microplastics in zebrafish (Danio rerio) and toxic effects in liver. Environmental science \& technology, 50(7), 4054-4060. 10.1021/acs.est.6b00183

Lucio, f. T., Magnoni, D. M., Vicentini, V. E. P.; \& Conte, H. (2019) disponibilidade e influência dos microplásticos nos seres vivos e ambiente: uma revisão. Conexão ci. 1, 14, $47-55$, formiga/mg. 10.24862/CCO.V14I1.908

Lusher, A. L., Hernandez-Milian, G., O'Brien, J., Berrow, S., O'Connor, I., \& Officer, R. (2015). Microplastic and macroplastic ingestion by a deep diving, oceanic cetacean: the True's beaked whale Mesoplodon mirus. Environmental Pollution, 199, 185-191. 10.1016/j.envpol.2015.01.023

Lwanga, E. H., Gertsen, H., Gooren, H., Peters, P., Salánki, T., van der Ploeg, M., Brosseling, E., Koelmans, A. A., \& Geissen, V. (2017). Incorporation of microplastics from litter into burrows of Lumbricus terrestris. Environmental Pollution, 220, 523-531. 10.1016/j.envpol.2016.09.096

Maab, S., Daphi, D., Lehmann, A., \& Rillig, M. C. (2017). Transport of microplastics by two collembolan species. Environmental Pollution, 225, 456-459. o10.1016/j.envpol.2017.03.009

Mak, C. W., Yeung, K. C. F., \& Chan, K. M. (2019). Acute toxic effects of polyethylene microplastic on adult zebrafish. Ecotoxicology and environmental safety, 182, 109442. 10.1016/j.ecoenv.2019.109442

Malafaia, G., de Souza, A. M., Pereira, A. C., Gonçalves, S., da Costa Araújo, A. P., Ribeiro, R. X., \& Rocha, T. L. (2020). Developmental toxicity in zebrafish exposed to polyethylene microplastics under static and semi-static aquatic systems. Science of The Total Environment, 700 , 134867. 10.1016/j.scitotenv.2019.134867

Mendoza, L. M. R., Karapanagioti, H., \& Álvarez, N. R. (2018). Micro (nanoplastics) in the marine environment: current knowledge and gaps. Current Opinion in Environmental Science \& Health, 1, 47-51. 10.1016/j.coesh.2017.11.004

Miller, M. E., Hamann, M., \& Kroon, F. J. (2020). Bioaccumulation and biomagnification of microplastics in marine organisms: A review and meta-analysis of current data. Plos one, 15(10), e0240792. 10.1371/journal.pone.0240792

Nelms, S. E., Duncan, E. M., Broderick, A. C., Galloway, T. S., Godfrey, M. H., Hamann, M., Lindeque, P. K., \& Godley, B. J. (2016). Plastic and marine turtles: a review and call for research. ICES Journal of Marine Science, 73(2), 165-181. 10.1093/icesjms/fsv 165

Neves, A. F., Paula, M. H., \& Anjos, P. H. R. (2016). Estudos interdisciplinares em ciências ambientais, território e movimentos sociais. São Paulo: Blucher. https://www.blucher.com.br/livro/detalhes/estudos-interdisciplinares-em-ciencias-ambientais-territorio-e-movimentos-sociais-1213

Ogonowski, M., Gerdes, Z., \& Gorokhova, E. (2018). What we know and what we think we know about microplastic effects-A critical perspective. Current Opinion in Environmental Science \& Health, 1, 41-46. 10.1016/j.coesh.2017.09.001

Olivatto, G. P., Carreira, R., Tornisielo, V. L., \& Montagner, C. C. (2018). Microplásticos: Contaminantes de preocupação global no Antropoceno. Revista Virtual de Química, 10(6), 1968-1989. 10.21577/1984-6835.20180125

Oliveira, M., Ribeiro, A., Hylland, K., \& Guilhermino, L. (2013). Single and combined effects of microplastics and pyrene on juveniles (0+ group) of the common goby Pomatoschistus microps (Teleostei, Gobiidae). Ecological indicators, 34, 641-647. 10.1016/j.ecolind.2013.06.019

Ory, N. C., Gallardo, C., Lenz, M., \& Thiel, M. (2018). Capture, swallowing, and egestion of microplastics by a planktivorous juvenile fish. Environmental Pollution, 240, 566-573. 10.1016/j.envpol.2018.04.093

Peres, R. M., Sanseverino, M. T. V., \& Faccini, L. S. (2001). Exposição a contaminantes ambientais durante a gestação e seus efeitos sobre a saúde fetal: uma revisão da literatura. Revista do HCPA \& Faculdade de Medicina da Universidade Federal do Rio Grande do Sul. Vol. 21, n. 3 (dez. 2001 ), p. 368-378. https://www.lume.ufrgs.br/handle/10183/163857

Pivokonsky, M., Cermakova, L., Novotna, K., Peer, P., Cajthaml, T., \& Janda, V. (2018). Occurrence of microplastics in raw and treated cience of the Total Environment, 643, 1644-1651. 10.1016/j.scitotenv.2018.08.102

Prata, J. C. (2018). Airborne microplastics: consequences to human health?. Environmental pollution, 234, 115-126. 10.1016/j.envpol.2017.11.043

Prata, J. C., da Costa, J. P., Lopes, I., Duarte, A. C., \& Rocha-Santos, T. (2020). Environmental exposure to microplastics: An overview on possible human health effects. Science of the Total Environment, 702, 134455. 10.1016/j.scitotenv.2019.134455

Revel, M., Châtel, A., \& Mouneyrac, C. (2018). Micro (nano) plastics: A threat to human health?. Current Opinion in Environmental Science \& Health, 1, 1723. 10.1016/j.coesh.2017.10.003

Ricklefs, R. E. (2003). A economia da natureza. Guanabara Koonga S.

Rillig, M. C., Ziersch, L., Hempel, S. (2017). Microplastic transport in soil by earthworms. Scientific reports, 7(1), 1-6. DOI:10.1038/s41598-017-01594-7 
Research, Society and Development, v. 10, n. 7, e54310716761, 2021

(CC BY 4.0) | ISSN 2525-3409 | DOI: http://dx.doi.org/10.33448/rsd-v10i7.16761

Rochman, C. M., Hoh, E., Kurobe, T., \& Teh, S. J. (2013). Ingested plastic transfers hazardous chemicals to fish and induces hepatic stress. Scientific reports, 3(1), 1-7. 10.1038/srep03263

Rochman, C. M., Kurobe, T., Flores, I., \& Teh, S. J. (2014). Early warning signs of endocrine disruption in adult fish from the ingestion of polyethylene with and without sorbed chemical pollutants from the marine environment. Science of the total environment, 493, 656-661. 10.1016/j.scitotenv.2014.06.051

Schuyler, Q., Hardesty, B. D., Wilcox, C., \& Townsend, K. (2014). Global analysis of anthropogenic debris ingestion by sea turtles. Conservation biology, 28(1), 129-139. 10.1111/cobi.12126

Schymanski, D., Goldbeck, C., Humpf, H. U., \& Fürst, P. (2018). Analysis of microplastics in water by micro-Raman spectroscopy: release of plastic particles from different packaging into mineral water. Water research, 129, 154-162. 10.1016/j.watres.2017.11.011

Simmonds, M. P. (2012). Cetaceans and marine debris: the great unknown. Journal of Marine Biology, 2012. 10.1155/2012/684279

Song, Y., Cao, C., Qiu, R., Hu, J., Liu, M., Lu, S., Shi, H., Raley-Susman, K. M., \& He, D. (2019). Uptake and adverse effects of polyethylene terephthalate microplastics fibers on terrestrial snails (Achatina fulica) after soil exposure. Environmental Pollution, 250, 447-455. 10.1016/j.envpol.2019.04.066

Vethaak, A. D., \& Leslie, H. A. (2016). Plastic debris is a human health issue. Environ. Sci. Technol. 50, 6825-6826. 10.1021/acs.est.6b02569

Von Moos, N., Burkhardt-Holm, P., \& Köhler, A. (2012). Uptake and effects of microplastics on cells and tissue of the blue mussel Mytilus edulis L. after an experimental exposure. Environmental science \& technology, 46(20), 11327-11335. 10.1021/es302332w

Yang, D., Shi, H., Li, L., Li, J., Jabeen, K., \& Kolandhasamy, P. (2015). Microplastic pollution in table salts from China. Environmental science \& technology, 49(22), 13622-13627. 10.1021/acs.est.5b03163

Zhu, D., Bi, Q. F., Xiang, Q., Chen, Q. L., Christie, P., Ke, X., Wu, L. H, \& Zhu, Y. G. (2018). Trophic predator-prey relationships promote transport of microplastics compared with the single Hypoaspis aculeifer and Folsomia candida. Environmental Pollution, 235, 150-154. 10.1016/j.envpol.2017.12.058 